\title{
Elastic properties of graphene flakes: Boundary effects and lattice vibrations
}

\author{
S. Bera, ${ }^{1,2}$ A. Arnold, ${ }^{2}$ F. Evers, ${ }^{1,2}$ R. Narayanan, ${ }^{3}$ and P. Wölfle ${ }^{1,2}$ \\ ${ }^{1}$ Institut für Theorie der Kondensierten Materie, Karlsruhe Institute of Technology, D-76128 Karlsruhe, Germany \\ ${ }^{2}$ Institut für Nanotechnologie, Karlsruhe Institute of Technology, D-76021 Karlsruhe, Germany \\ ${ }^{3}$ Department of Physics, Indian Institute of Technology Madras, Chennai 600036, India
}

(Received 23 March 2010; revised manuscript received 4 October 2010; published 30 November 2010)

\begin{abstract}
We present a phenomenological theory together with explicit calculations of the electronic ground-state energy, the surface contribution, and the elastic constants ("Lamé parameters," i.e., Poisson ratio, Young's modulus) of graphene flakes on the level of the density-functional theory employing different standard functionals. We observe that the Lamé parameters in small flakes can differ from the bulk values by $30 \%$ for hydrogenated zigzag edges. The change results from the edge of the flake that compresses the interior. When including the vibrational zero-point motion, we detect a decrease in the bending rigidity, $\kappa$, by $\sim 26 \%$. The vibrational frequencies flow with growing $N$ due to the release of the edge-induced compression. We calculate the corresponding Grüneisen parameters and find good agreement with previous authors.
\end{abstract}

DOI: 10.1103/PhysRevB.82.195445

PACS number(s): 81.05.ue, 62.20.D-, 62.25.-g, 63.22.Rc

\section{INTRODUCTION}

Since its fabrication has become technologically feasible, ${ }^{1}$ graphene has been in the focus of frontier research. ${ }^{2-4}$ One of its most celebrated properties are its massless low-energy excitations 5,6 ("Dirac fermions"), which emanate from the symmetries of the honeycomb lattice. The electronic properties of graphene flakes are quite different from bulk graphene due to the finite size and the presence of edges. ${ }^{7,8}$ In particular, calculations suggest that the zigzag edges of graphene nanoribbons (quasi-one-dimensional) have two flat bands at the Fermi energy ${ }^{9,10}$ that introduce magnetism. ${ }^{11-18}$ Recent theoretical studies on zigzag-edged graphene flakes also confirm a tendency toward edge magnetism. ${ }^{17-20}$ Thus this gives an added motivation for fabricating graphene-based nanostructures. ${ }^{21-26}$ The fabrication of such structures with well-defined edges still poses a considerable technological challenge. Therefore, only very few experiments with structures exhibiting zigzag edges have been reported; ${ }^{27-29}$ a detailed investigation of the edge physics still needs to be done.

An increased interest in the elastic properties of graphene has developed recently. ${ }^{21,30-43}$ This is, for instance, because experiments suggest that graphene samples exhibit a corrugated structure $28,44-46$ ("ripples") even at relatively low temperatures. Their origin is thought to be due to residual elastic strain produced by the experimental preparation technique. ${ }^{47}$ Another motivation comes from exploring the feasibility of strain engineering the electronic band structure ${ }^{48}$ ("pseudomagnetic" fields).

Also for elastic properties, edge effects can be highly relevant. Hence studies of flake elastic properties are interesting in their own right. Namely, there is an intimate relation between the electronic structure and the atomic geometry of graphene. For example, the electronic spectrum of a certain class of armchair graphene nanoribbons is reported to acquire a spectral gap due to an edge-induced lattice dimerization along the transport direction. ${ }^{13}$

In our study we investigate the ground-state energy, the elastic properties, and the phonon spectrum of $N \times N$ graphene sheets ("flakes") as displayed in Fig. 1 using the density-functional theory (DFT). We show that the different chemical nature of $\mathrm{C}-\mathrm{C}$ bonds at the hydrogenated edge as compared to the bulk leads to a nearly homogenous isotropic compression, i.e., strain. As a consequence, the average C-C distance in a $3 \times 3$ flake is reduced by a substantial amount, $0.3 \%$; for comparison, strain as achieved in typical pressure experiments does not usually exceed values $\sim 1 \%$. ${ }^{34,49,50}$

The presence of the surface-induced strain leaves various traces in the flakes' interior observables. (a) The flakes elastic constants, i.e., the Lamé parameters, are enhanced as compared to the bulk case. For isotropic strain in smallest flakes $(3 \times 3)$, the (inverse) compressibility $\mu+\lambda$ [for a precise definition see below, Eq. (2)] increases by $30 \%$; for shear forces the increase is even bigger, almost a factor of 2 . (b) Under bond compression the interatomic forces typically increase so that even the short-wavelength vibrations, in particular, the optical phonons, exhibit a "blueshift" of their frequencies with decreasing flake sizes. This "flow" can be seen in the variation in the Raman spectra with strain $^{27,49,51-54}$ and can be described in the standard manner by Grüneisen parameters. The values that we find here of Grüneisen parameters, agree reasonably well with previous reports. ${ }^{49,52,55}$

Even though one might suspect, that our topics have already been dealt with extensively in the literature, ${ }^{54-58}$ a detailed investigation of the elastic properties of nanoflakes is yet to be done; this refers, in particular, to an analysis of edge and finite-size effects of hydrogenated zigzag flakes which we perform in this work.

\section{GROUND-STATE ENERGY OF HOMOGENOUS, PLANAR FLAKES}

Quite generally, the total energy of a graphene flake such as the one depicted in Fig. 1 is a sum of local contributions. In principle, these may be thought of as contributions per bond or per atom. The latter perspective has been worked out in the Appendix. Here, we will focus on a representation in terms of bond energies valid for flakes with a homogenous C-C distance, $d$ 


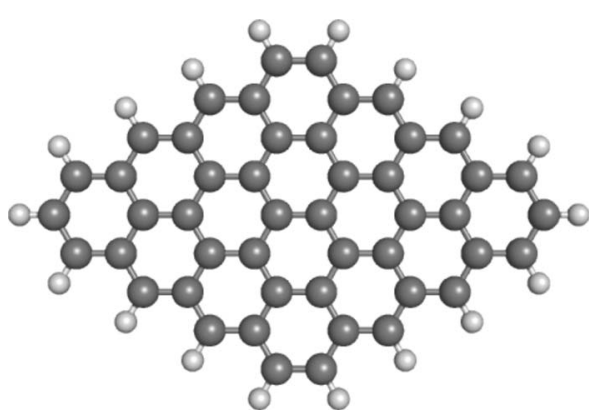

FIG. 1. Geometry of the $N \times N$ hydrogenated graphene flakes(here $N=4$ ), that we have used for the density-functional calculations.

$$
F_{N}(d)=N_{\mathrm{i}} \Psi(d)+N_{\mathrm{e}} \psi(d)+N_{\mathrm{c}} \psi^{\mathrm{c}}(d)+\frac{\phi^{\mathrm{e}}(d)}{N_{\mathrm{e}}}+\frac{\phi^{\mathrm{i}}(d)}{N_{\mathrm{i}}},
$$

where $N_{\mathrm{i}}$ denotes the number of internal C-C bonds with an associated binding energy $\Psi, N_{\mathrm{e}}$ denotes the number of edge located C-C bonds with energy $\psi$, and $\psi^{c}$ includes the corner contributions, where $N_{\mathrm{c}}$ is the number of bonds linking the ten corner atoms, $N_{\mathrm{c}}=6$ in Fig. 1 . The coefficients $\phi^{\text {e, i }}$ are further expansion parameters. The binding energy per $\mathrm{C}-\mathrm{C}-\mathrm{CH}$ edge group is close to $2 \psi$ but not identical to it. For instance, $\psi$ also includes corrections of internal bonds, that still "feel" the presence of the surface. Similarly, $N_{c} \psi^{c}$ is approximating the binding energy of the corner groups (two $\mathrm{HC}-\mathrm{CH}$ groups and two C-CH-C groups).

We mention that the representation in Eq. (1) is slightly simplified in the following sense. In general, the boundary (shape) of a given flake, e.g., as depicted in Fig. 1 does not share the hexagonal symmetry of the honeycomb lattice. For this reason, in flakes with a fully relaxed atomic structure bond lengths and bond angles are not strictly all the same. Our DFT calculations indicate, however, that such distortions, though clearly detectable, give only small corrections to those phenomenological parameters that we are mostly interested in.

The continuum theory of two-dimensional membranes has been devised for an inhomogeneous flake with neighboring bonds exhibiting slowly (in space) varying bond distances, $d(\mathbf{r})$, and angles. In this formulation the elastic energy is represented by the functional, 59

$$
\begin{aligned}
E= & \frac{\kappa}{2} \int_{\mathcal{A}} d^{2} \mathbf{r}\left(\nabla^{2} h\right)^{2}+\frac{\mu+\lambda}{2} \int_{\mathcal{A}} d^{2} \mathbf{r}\left(u_{x x}+u_{y y}\right)^{2} \\
& +\frac{\mu}{2} \int_{\mathcal{A}} d^{2} \mathbf{r}\left[4 u_{x y}^{2}+\left(u_{x x}-u_{y y}\right)^{2}\right] .
\end{aligned}
$$

The flake coordinates are given with respect to a planar reference state with area $\mathcal{A}$, that lives in the $\mathbf{r}=(x, y)$ plane; accordingly, the in-plane coordinates constitute the displacement vector, $\mathbf{u}(x, y)$, that measures the translation of each membrane point $(x, y, z)$ with respect to the reference state. The out-of-plane distortions define the height field $h(x, y)$; for the planar case $h=0$. $\mathbf{u}$ and $h$ together constitute the strain tensor $(i, j=x, y)$,

$$
u_{i j}=\frac{1}{2}\left[\partial_{i} u_{j}+\partial_{j} u_{i}+\partial_{i} h \partial_{j} h+\cdots\right],
$$

where (nonlinear) higher order gradient terms have been neglected. It is clear that the form of $u_{i j}$ depicted in Eq. (3) is a symmetric construction with respect to the spatial derivatives of $\mathbf{u}$ ensuring, e.g., invariance under in-plane rotations. Such infinitesimal rotations correspond to $\mathbf{u} \propto(y,-x)$ and hence the strain must be invariant under such displacements $\mathbf{u}$.

The total elastic energy Eq. (2) is a sum over contributions which resemble local oscillators in the membrane plane. The first term is proportionate to the curvature $\nabla^{2} h$ and introduces the bending rigidity $\kappa$. It describes the energy cost for bending the membrane without changing the bond lengths or in-plane bond angles. ${ }^{60}$ The Lamé parameters, $\lambda$ and $\mu$, appearing in the second and third terms of Eq. (2) describe the in-plane rigidity.

For homogenous, planar membranes the elastic theory Eq. (2) may be considered as a continuum approximation to Eq. (1) which does not make explicit reference to boundary terms. Edges are accounted for only in the boundary conditions and (possibly) in a dependency of the Lamé parameters on the position with respect to the edge. Usually not included in Eq. (2) is the fact that this spatial dependency supports long-range terms, $\sim 1 /$ flake size. They modify the Lamé parameters appearing in Eq. (2) even inside the flake's interior.

\section{Phenomenological parameters}

\section{Isotropic strain}

In order to illustrate the cooperative effect between surface and bulk, we consider an expansion of Eq. (1) in terms of the variable $\varepsilon=\left(d-d_{0}\right) / d_{0} ; \varepsilon$ quantifies the strain inside the flake. The bulk energy per bond has an expansion,

$$
\Psi=\Psi_{0}+\frac{1}{2} \Psi_{2} \varepsilon^{2}+\frac{1}{6} \Psi_{3} \varepsilon^{3}+\frac{1}{24} \Psi_{4} \varepsilon^{4}+\cdots,
$$

where the bulk bond length $d_{0}$ is to be determined at $N_{\mathrm{i}, \mathrm{e}}$ $\rightarrow \infty$. The surface energy may also be expanded about a minimum bond length, $d_{0}^{\mathrm{e}}$, but, in general, $d_{0}^{\mathrm{e}} \neq d_{0}$. After all, in the limit $N_{\mathrm{i}, \mathrm{e}} \rightarrow \infty$, just the first term in Eq. (1) contributes to the energy per area and therefore $d_{0}$ needs to minimize $\Psi$, only. Hence, we introduce the relative deviation of surface and bulk optimal bond lengths, $\mathfrak{d}=\left(d_{0}-d_{0}^{\mathrm{e}}\right) / d_{0}$, so that we have an expansion

$$
\begin{aligned}
\psi & =\bar{\psi}_{0}+\frac{1}{2} \bar{\psi}_{2}(\varepsilon+\mathfrak{d})^{2}+\frac{1}{6} \bar{\psi}_{3}(\varepsilon+\mathfrak{d})^{3}+\frac{1}{24} \bar{\psi}_{4}(\varepsilon+\mathfrak{d})^{4} \cdots \\
& \equiv \psi_{0}+\psi_{1} \varepsilon+\frac{1}{2} \psi_{2} \varepsilon^{2}+\frac{1}{6} \psi_{3} \varepsilon^{3}+\cdots
\end{aligned}
$$

where the coefficients in the second line are defined in terms of the expansion the line before. The elastic properties of the flake are determined by the expansion parameters $\Psi_{2,3,4}, \psi_{1,2,3}$.

At any finite value of $N_{\mathrm{i}, \mathrm{e}}$, optimization must also include the boundary (i.e., surface) terms and therefore the optimal value of $\varepsilon, \varepsilon_{N}$, is nonvanishing in this case; specifically, 


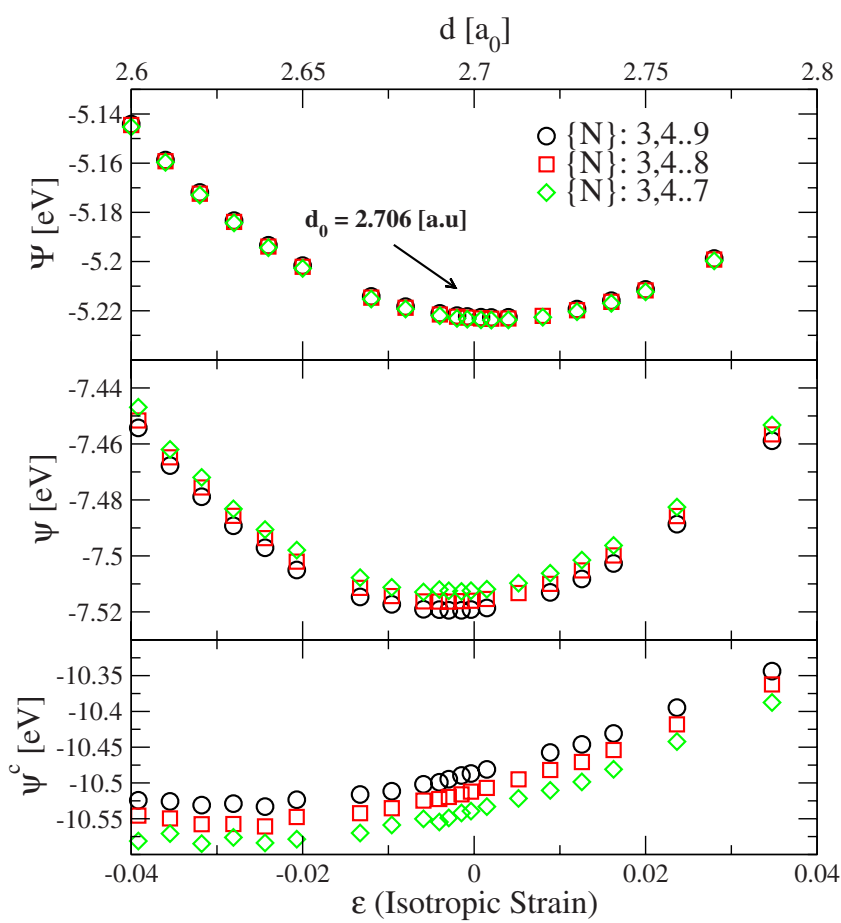

FIG. 2. (Color online) Bulk $(\Psi)$, surface (edge, $\psi$ ), and corner $\left(\psi^{c}\right)$ energy per carbon bond in the graphene flakes, Fig. 1, calculated with density-functional theory (BP86 functional). Data based on the evaluation of three sets of flake sizes ranging from $\{N\}$ $=3, \ldots, 9$, and a five-parameter fit to Eqs. (1) and (11) per $d$ values. $\left(a_{0}:=0.529 \AA\right.$ A. $)$

$$
\varepsilon_{N}=-\frac{\psi_{1}}{\Psi_{2}} \frac{N_{\mathrm{e}}}{N_{\mathrm{i}}} \approx-\mathfrak{d} \frac{\bar{\psi}_{2}}{\Psi_{2}} \frac{N_{\mathrm{e}}}{N_{\mathrm{i}}}
$$

In order to calculate the feedback of this shift into the elastic parameters, we expand $F$ in the vicinity of its minimum, $\varepsilon_{N}$, to the fourth order in $\varepsilon-\varepsilon_{N}$. Recalling that this corresponds to a strain $\mathbf{u}(\mathbf{x})=\varepsilon \mathbf{x}$ we can compare the result with Eq. (2) and thus find to leading order in $N_{\mathrm{e}} / N_{\mathrm{i}}$

$\mu+\lambda=\frac{1}{4}\left(\Psi_{2}+\frac{N_{\mathrm{e}}}{N_{\mathrm{i}}} \psi_{2}\right)+\frac{1}{4} \Psi_{3} \varepsilon_{N}+\frac{\varepsilon}{12}\left(\Psi_{3}+\frac{N_{\mathrm{e}}}{N_{\mathrm{i}}} \psi_{3}+\Psi_{4} \varepsilon_{N}\right)$.

The first term on the rhs of expression (7) simply accounts for the separate, additive contributions of bulk and surface (i.e., edge) energies. The edge contribution, that appears here, could formally be accounted for in a generalized version of Eq. (2) where one adds a boundary term. Similarly, by allowing for a dependency of the Lamé parameters on strain itself, one could also include additive anharmonic effects, second bracket first two terms. In either case, the phenomenological parameters that enter the conventional elastic theories of the continuum are the same for each flake size and shape. Adapting these parameters to each flake geometry separately is not foreseen in this framework

The interesting pieces in $\varepsilon_{N}$ are the terms, which mix the surface and bulk parameters: $\Psi_{3} \varepsilon_{N} \sim \psi_{1} \Psi_{3} / \Psi_{2}$. They encode the "cooperative" effect between boundary induced strain
TABLE I. Minimum C-C bond length as extracted from bulk energy and surface energy correspondingly (see Fig. 2, upper and middle panels). The distance in $d_{0}$ and $d_{o}^{\mathrm{e}}$ leads to a nearly homogenous pressure on the flake that modifies elastic and electronicstructure properties. Data are shown for three different functionals used in DFT calculation.

\begin{tabular}{lcc}
\hline \hline & $\begin{array}{c}d_{0} \\
(\AA)\end{array}$ & $\begin{array}{c}d_{0}^{\mathrm{e}} \\
(\AA)\end{array}$ \\
\hline BP86 & $1.432 \pm 0.002$ & $1.427 \pm 0.001$ \\
B3LYP & $1.426 \pm 0.001$ & $1.421 \pm 0.002$ \\
PBE & $1.431 \pm 0.001$ & $1.426 \pm 0.005$ \\
\hline \hline
\end{tabular}

and bulk anharmonicities. It is due to them, that the flakes elastic parameters need to be adjusted in principle for every geometry separately.

\section{Shear strain}

An analogous analysis as for the isotropic strain also applies to shear forces. The expansion is even in the shear strain $\mathbf{u}=\left(0, \varepsilon_{\mathrm{s}} x\right)$

$$
\begin{gathered}
\Psi=\Psi_{0}+\frac{1}{2} \widetilde{\Psi}_{2} \varepsilon_{\mathrm{s}}^{2}+\frac{1}{24} \widetilde{\Psi}_{4} \varepsilon_{\mathrm{s}}^{4}+\cdots, \\
\psi=\psi_{0}+\frac{1}{2} \widetilde{\psi}_{2} \varepsilon_{\mathrm{s}}^{2}+\frac{1}{24} \widetilde{\psi}_{4} \varepsilon_{\mathrm{s}}^{4}+\cdots .
\end{gathered}
$$

The new expansion parameters, $\widetilde{\Psi}_{i}, \widetilde{\psi}_{i}, i=2,4, \ldots$, are, in general, dependent on the flake geometry. Again, a comparison to Eq. (2) yields

$$
\mu=\widetilde{\Psi}_{2}+\widetilde{\psi}_{2}+\frac{1}{12} \varepsilon_{\mathrm{s}}^{2}\left(\widetilde{\Psi}_{4}+\widetilde{\psi}_{4}\right) .
$$

Here, the surface and bulk energies give strictly additive contributions, and a cooperative effect does not emerge.

\section{DENSITY-FUNCTIONAL CALCULATIONS}

\section{A. Method}

In Fig. 1 we display the geometry of the $N \times N$-graphene flake that is employed in our calculations: $N_{\mathrm{i}}=(N-1)(3 N$ $-1)$ and $N_{\mathrm{e}}=8(N-1)$. Electronic structure calculations have been performed for a given atomic configuration (C-C distance, flake geometry, etc.) on the basis of the densityfunctional theory as implemented in the quantum chemistry package TURBOMOLE. ${ }^{61}$ We are comparing GGA functionals [BP86 (Refs. 62 and 63) and PBE (Refs. 64 and 65)] with a hybrid functional [B3LYP (Ref. 66)] and use a minimal basis set [SVP (Ref. 67)]. Specifically, we are working at zero temperature and approximate the ground-state energy, Eq. (1), by the DFT estimate for the total binding energy of the flake

$$
F_{\mathrm{el}}(N, d):=E_{\mathrm{el}}(N, d)-E_{\text {free }}(N)
$$

with 
TABLE II. Bulk-energy coefficients as defined in Eq. (4). These coefficients are extracted from fitting Eq. (4) to the data in Fig. 2, upper panel.

\begin{tabular}{lccc}
\hline \hline & $\begin{array}{c}-\Psi_{0} \\
(\mathrm{eV})\end{array}$ & $\begin{array}{c}\frac{1}{2} \Psi_{2} \\
(\mathrm{eV})\end{array}$ & $\begin{array}{c}-\frac{1}{6} \Psi_{3} \\
(\mathrm{eV})\end{array}$ \\
\hline BP86 & $5.223 \pm 0.001$ & $46.301 \pm 0.082$ & $128.3 \pm 2$ \\
B3LYP & $5.008 \pm 0.001$ & $47.7 \pm 0.4$ & $154 \pm 32$ \\
PBE & $5.373 \pm 0.004$ & $45.57 \pm 0.43$ & $187 \pm 25$ \\
\hline \hline
\end{tabular}

$$
E_{\text {free }}(N)=N_{\mathrm{H}} E_{\mathrm{H}}+N_{\mathrm{C}} E_{\mathrm{C}},
$$

where $E_{\mathrm{H} / \mathrm{C}}$ denote the DFT energies of a free charge neutral hydrogen/carbon atom and $N_{\mathrm{H} / \mathrm{C}}$ denotes the number of hydrogen/carbon atoms in the flake.

\section{B. Results and discussion}

\section{Isotropic strain}

A sequence of DFT calculations has been performed for $N=3, \ldots, 9$ and different values of the C-C distance, $d$. For each distance, $F_{\mathrm{el}}(N, d)$ has been calculated. In order to extract the expansion coefficients of Eq. (1), $\Psi(d), \psi(d), \psi^{\mathrm{e}}(d), \phi^{\mathrm{e}}(d), \phi^{\mathrm{i}}(d)$, we have performed fiveparameter fits on sets of raw DFT data. These fits were applied to three data sets consisting of $\{N\}=3, \ldots, 7,\{N\}$ $=3, \ldots, 8$, and $\{N\}=3, \ldots, 9$. The results for the surface, bulk, and corner energy have been displayed in Fig. 2. The scatter between the fitting parameters belonging to different data sets is relatively small, which illustrates the stability of the fit.

The lattice constant of bulk graphene is estimated from the minimum position of $\Psi(d)$ Fig. 2, upper panel as $d_{0}$ $=2.706 a_{0}$, where $a_{0}=0.529 \AA$ denotes the Bohr radius. Comparing this position to the minimum of the edge (surface) energy, Fig. 2, center panel, $d_{0}^{\mathrm{e}}=2.694 a_{0}$, we find $\mathfrak{d}$ $=0.44 \%$. This indicates clearly the compression of the C-C bond length near the edge. The shift of the minimum position to lower values becomes even more pronounced near the corners, i.e., in $\psi^{\varepsilon}(d)$, see Fig. 2, lower panel.

To obtain also the other phenomenological parameters, a second (polynomial) fit of the traces $\Psi(d), \psi(d)$, Fig. 2, according to Eqs. (4) and (5) has been performed; all fitting parameters are summarized in Tables I-III.

When fitting the raw data to get $\Psi, \psi, \psi^{\mathrm{c}}$ the terms in $1 / N_{\mathrm{i}, \mathrm{e}}$ could not be neglected for the system sizes that we

TABLE III. Edge (surface) coefficients as defined in Eq. (5). These coefficients are extracted from fitting Eq. (5) to the data in Fig. 2, middle panel

\begin{tabular}{lcccc}
\hline \hline & $\begin{array}{c}-\psi_{0} \\
(\mathrm{eV})\end{array}$ & $\begin{array}{c}\psi_{1} \\
(\mathrm{eV})\end{array}$ & $\begin{array}{c}\frac{1}{2} \psi_{2} \\
(\mathrm{eV})\end{array}$ & $\begin{array}{c}-\frac{1}{6} \psi_{3} \\
(\mathrm{eV})\end{array}$ \\
\hline BP86 & $7.515 \pm 0.006$ & $0.285 \pm 0.015$ & $44.93 \pm 0.43$ & $113 \pm 19$ \\
B3LYP & $7.187 \pm 0.006$ & $0.261 \pm 0.015$ & $45.6 \pm 2.2$ & \\
PBE & $7.63 \pm 0.01$ & $0.308 \pm 0.011$ & $47.5 \pm 1.1$ & \\
\hline \hline
\end{tabular}

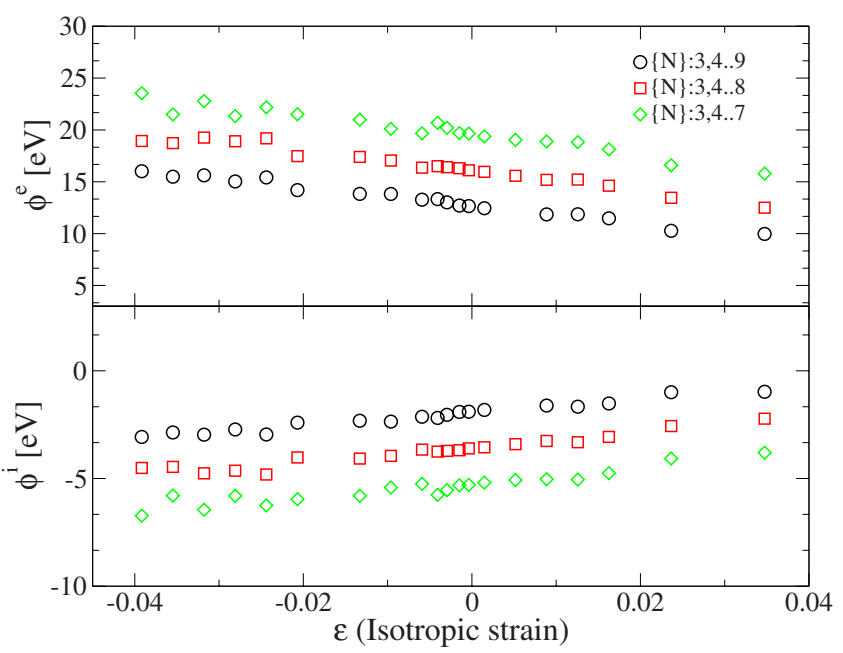

FIG. 3. (Color online) Dependency of the amplitudes $\phi^{\text {i,e }}$ describing the corrections in $1 / N_{\mathrm{e}, \mathrm{i}}$ to the binding energy $\Delta F_{\mathrm{el}}(N)$. Data were obtained by the five-parameters fit, already underlying the traces shown in Fig. 2.

considered. The corresponding amplitudes are displayed in Fig. 3. Unlike it was the case with the previous data, Fig. 2, the amplitudes $\phi^{\mathrm{i}, \mathrm{e}}$ of the $1 / N_{\mathrm{i}, \mathrm{e}}$ corrections still exhibit a considerable variation with increasing system size, which is due to even order terms that have been neglected in the expansion Eq. (1). Interestingly, while the magnitude of $\phi^{\mathrm{i}, \mathrm{e}}(\varepsilon)$ is still shifting the slope and perhaps also the sign of the two functions have converged, already. Under this assumption we may conclude that both amplitudes flow closer to zero values when $\varepsilon$ increases. This behavior is compatible with the simple expectation that the main effect incorporated in the $1 / N_{\mathrm{i}, \mathrm{e}}$ corrections is the discreteness of the flake's electronic spectrum with level spacings $\Delta_{\mathrm{i}, \mathrm{e}}$ for bulk and surface modes. With increasing $\varepsilon$ the bandwidth decreases and so do $\Delta_{\mathrm{i}, \mathrm{e}}$ and $\phi^{\mathrm{i}, \mathrm{e}}$.

In the Appendix we have repeated this analysis for a representation of the free energy as a sum of atomic contributions, Eq. (A1). The results are equivalent, of course, but the detailed comparison of the results based on our phenomenological theory with earlier findings for bulk systems, which we have relegated there, is simplified in this way. In a nutshell, our extrapolation scheme recovers the known values for the surface free energy (per Angstrom) and the edge stress with excellent accuracy, see Table VII and below Eq. (A4).

\section{Shear strain}

A largely analogous method as was adopted for the isotropic strain has also been applied for shear forces. In this case, the convergence of the DFT calculations turned out to be considerably more difficult so that the investigated system sizes range from $\{N\}=3, \ldots, 7$, only. From our fitting procedure we could determine the response of the bulk energy and surface energy to the shear strain, $\varepsilon_{\mathrm{s}}$, as shown in Fig. 4. The parameters entering Eqs. (8) and (9) can be extracted and are listed in Table IV. 


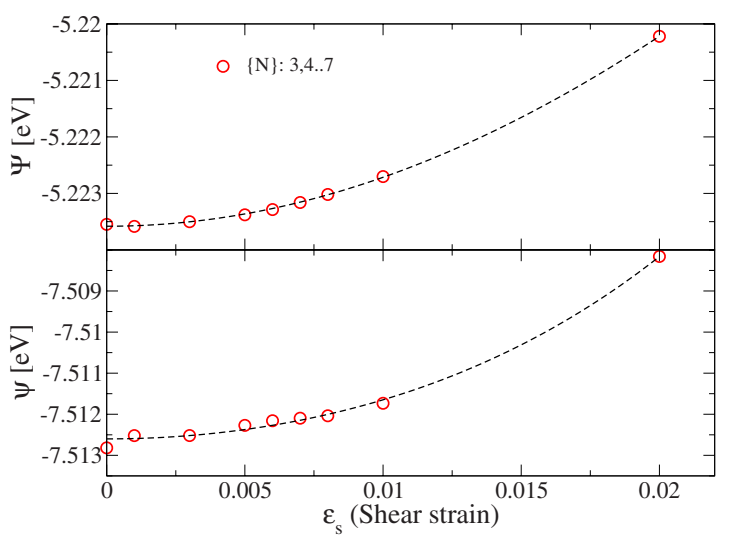

FIG. 4. (Color online) Change in the energy when applying a shear strain in graphene flakes. Upper panel shows the change in bulk energy per interior $\mathrm{C}-\mathrm{C}$ bond; lower panel exhibits change in surface (edge) energy per C-C bond. Data sets are for system sizes $\{N\}=3, \ldots, 7$ and extracted from an expression analogous for shear to Eqs. (1) and (11). The lines indicate the polynomial fit according to Eqs. (8) and (9) with parameter given in Table IV.

\section{FLAKE ELASTIC PROPERTIES}

In the previous section, the focus was on the behavior of the energy on the flake size under isotropic and shear strains. In this section, we discuss and illustrate what our previous findings imply for the elastic properties of a single flake with a fixed size, $N$. Partly, we are considering the same set of data again, but now plotting observables directly for $N$ fixed.

\section{A. Homogenous isotropic strain}

Figure 5 shows, how the excess energy per unit cell grows under increasing strain for different flake sizes $N$. It is readily seen from this plot, that there is a shift of the equilibrium lattice constant $d(N)$ to smaller values. In the light of the previous section, this shift is the expected consequence of the surface-induced strain $\varepsilon_{N}$. The inset shows the scaling with $N_{\mathrm{e}} / N_{\mathrm{i}}$.

In addition, we also extract the flake elastic constant $\mu$ $+\lambda$ from the parabolic shape of the curves, Fig. 5. To this end, we replot the data in Fig. 6 left, so as to highlight the curvature and its strain dependency. On the basis of Eq. (7) we can conclude that the offset of the curves is a consequence of (a) the presence of the surface and the extra energy required for its compression [term $N_{\mathrm{e}} / N_{\mathrm{i}}$ in Eq. (7)] and (b) the feedback of the surface strain $\varepsilon_{N}$ into the bulk C-C distance. Extrapolating the zero-strain values into the limit, $N$ $\rightarrow \infty$, we find $\mu_{\mathrm{el}}+\lambda_{\mathrm{el}} \approx 71$ per benzene ring, see Table $\mathrm{V}$,

TABLE IV. Bulk- and surface-shear-energy coefficients as extracted from fitting Eq. (8) [Eq. (9)] to the data in Fig. 4 upper panel (lower panel).

\begin{tabular}{ccccc}
\hline \hline & $\begin{array}{l}\frac{1}{2} \widetilde{\Psi}_{2} \\
(\mathrm{eV})\end{array}$ & $\begin{array}{c}-\frac{1}{24} \widetilde{\Psi}_{4} \\
(\mathrm{eV})\end{array}$ & $\begin{array}{c}\frac{1}{2} \widetilde{\psi}_{2} \\
(\mathrm{eV})\end{array}$ & $\begin{array}{c}\frac{1}{24} \tilde{\psi}_{4} \\
(\mathrm{eV})\end{array}$ \\
\hline BP86 & $8.4 \pm 0.4$ & $871 \pm 50 \%$ & $9 \pm 3.3$ & $5237 \pm 60 \%$ \\
\hline \hline
\end{tabular}

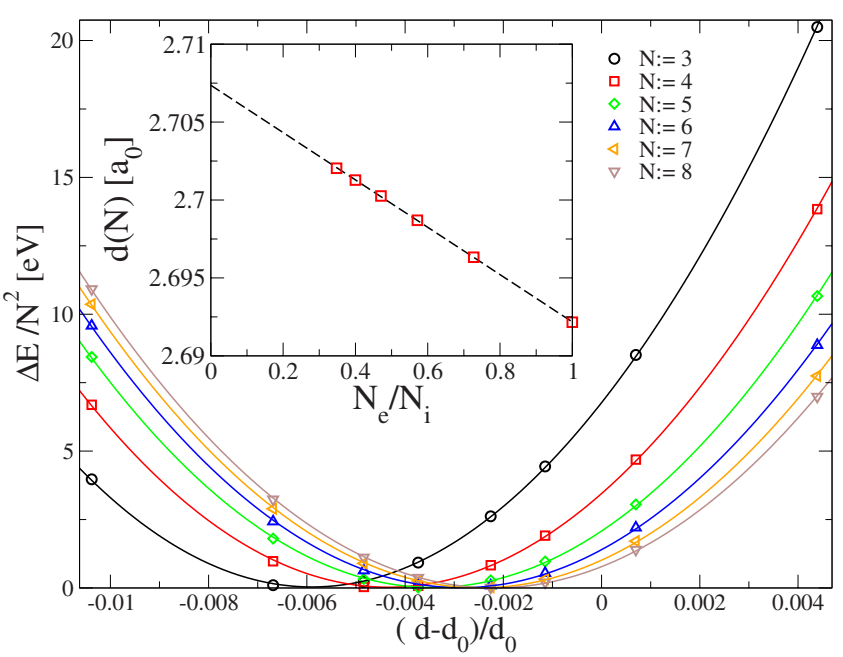

FIG. 5. (Color online) Excess energy $\Delta E$ per unit cell generated by rescaling all bond length, $d$, (homogeneous, isotropic strain). $\Delta E$ exhibits flow of the equilibrium bond length with the linear flake size $N$. Main panel: $\Delta E$ over bond length $d$ employed in the simulation. $d$ is measured relative to the bulk bond length. The lines serve as a guide to the eyes. Inset: extrapolating the equilibrium bond length $d(N)$ into the bulk limit: $d_{0}=2.707 \pm 0.001$.

matching well our result $\Psi_{2} / 4 \approx 23.5$ per (bulk) bond derived in the previous section, Table II, since each ring contributes three bonds. This check is displayed in Fig. 6, right. The plot also reveals that the deviation of elastic parameters from their bulk values in small flakes may not be very small. For our smallest flakes, $N=3$ it reaches almost $30 \%$.

Additional information can be extracted from Fig. 6 left, about anharmonicities which manifest themselves in the slope of the curves displayed. This prefactor of the anharmonic term (linear in $\varepsilon$ ) in Eq. (7) admits the following interpretation. The slope changes with increasing $N$ since the

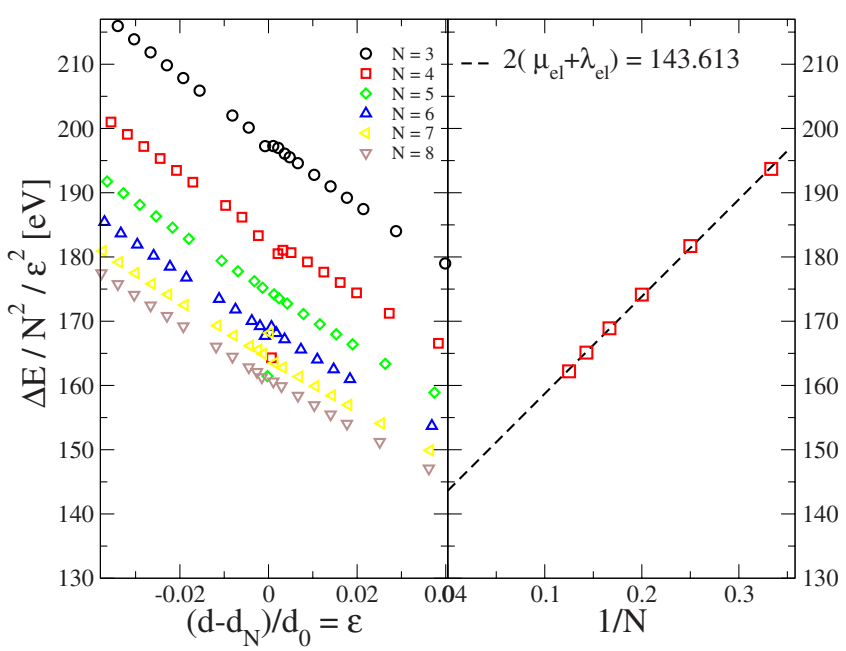

FIG. 6. (Color online) Estimating the sum of the Lamé parameters $\mu_{\mathrm{el}}+\lambda_{\mathrm{el}}$ and the boundary correction (offset of traces) from $\Delta E$ displayed in the previous Fig. 5. Left: data for curvature exhibit a slope which indicates the linear dependency of the Lamé parameter $\lambda_{\mathrm{el}}$ on strain. (Linear terms in $\mu_{\mathrm{el}}$ do not appear, see Fig. 9.) Right: extrapolating the curvature at $\varepsilon=0$ into the bulk limit. 
TABLE V. Comparison of C-C-bond distance in bulk graphene, elastic constants, Poisson ratio $[\nu=\lambda /(2 \mu+\lambda)]$, and Young's modulus $\left[Y=2(\mu+\lambda)(1-\nu) / \mathcal{A}_{\text {ring }}\right]$ as extracted from Figs. 5-7, respectively, by extrapolating the values in bulk limit $(N \rightarrow \infty)$ with previous works. Data are shown for three different functionals we used in DFT calculation. The area of a benzene ring is estimated as $\mathcal{A}_{\text {ring }}=3 \sqrt{3} d_{0}^{2} / 2$.

\begin{tabular}{|c|c|c|c|c|c|}
\hline & $\begin{array}{l}d_{0} \\
(\AA)\end{array}$ & $\begin{array}{c}\mu_{\mathrm{el}}+\lambda_{\mathrm{el}} \\
(\mathrm{eV})\end{array}$ & $\begin{array}{c}\mu_{\mathrm{el}} \\
(\mathrm{eV})\end{array}$ & $\nu$ & $\begin{array}{c}Y \\
(\mathrm{~N} / \mathrm{m})\end{array}$ \\
\hline BP86 & $1.432 \pm 0.001$ & $70.715 \pm 0.011$ & $50.95 \pm 0.01$ & 0.162 & 356.23 \\
\hline B3LYP & $1.427 \pm 0.001$ & $71.21 \pm 0.12$ & & & \\
\hline PBE & $1.431 \pm 0.002$ & $69.027 \pm 0.012$ & & & \\
\hline \multirow[t]{6}{*}{ Prev. calc. } & 1.42 (Refs. 37 and 70) & 66.571 (Ref. 37) & 49.45 (Ref. 37) & & 346 (Ref. 37) \\
\hline & 1.41 (Ref. 43$)$ & & & 0.173 (Ref. 31) & 307 (Ref. 41) \\
\hline & 1.45 (Ref. 71) & & & 0.16 (Ref. 71) & 336 (Ref. 71) \\
\hline & & & & 0.31 (Ref. 39) & 312 (Ref. 39) \\
\hline & & & & 0.149 (Ref. 72) & 345 (Ref. 72) \\
\hline & & & & 0.179 (Ref. 73) & 341 (Ref. 73) \\
\hline Expt. (Graphene) & & & & & 342 (Ref. 34) \\
\hline Expt. (Graphite) & 1.421 (Ref. 74) & & & 0.165 (Ref. 75) & $371(\text { Ref. } 76)^{\mathrm{a}}$ \\
\hline & 1.422 (Ref. 76) & & & & \\
\hline
\end{tabular}

${ }^{\mathrm{a}}$ Assuming graphene thickness $0.335 \mathrm{~nm}$.

contributions of the surface $\left(\psi_{3}\right.$ term $)$ and the surfaceinduced bulk compression $\left(\Psi_{4} \varepsilon_{N}\right.$ term) diminish. A nonvanishing value of $\Psi_{3} / 12$ for the slope will remain however even in the bulk limit.

\section{B. Shear strain}

Following the same strategy as we did before with Fig. 6, we plot in Fig. 7 the excess energy $\Delta E_{\mathrm{s}}$ induced by pure shear strain, $\mathbf{u}(\mathbf{x})=\varepsilon_{\mathrm{s}}(0, x)$. Again, the plot emphasizes the

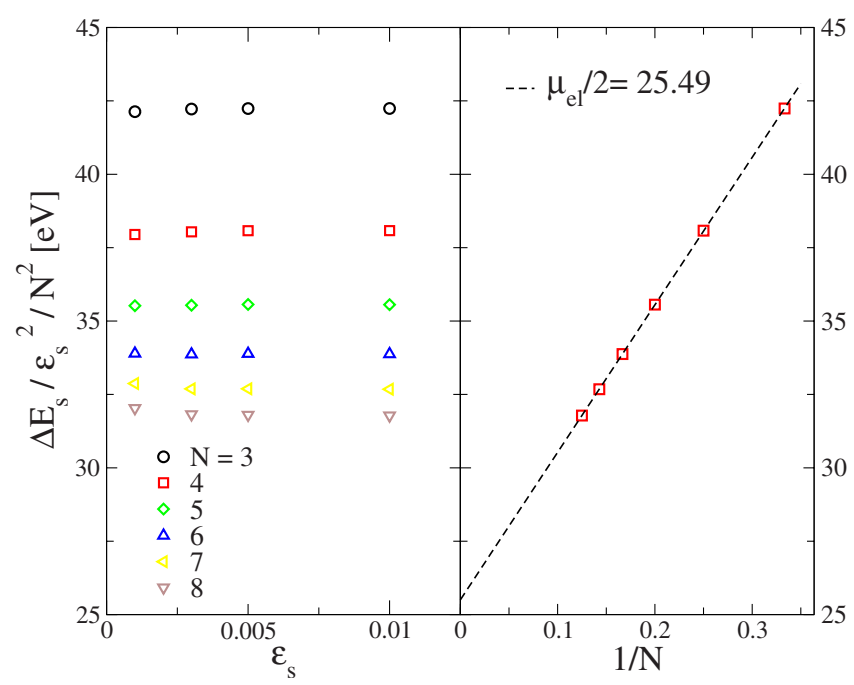

FIG. 7. (Color online) Estimate for Lamé parameter $\mu_{\mathrm{el}}$ determined from the excess energy $\Delta E_{\mathrm{s}}$ per unit cell under pure shear strain with strength $\varepsilon_{\mathrm{s}}$. (Procedure similar to previous Fig. 6.) Left panel: dependency of curvature of $\Delta E_{\mathrm{s}}\left(\varepsilon_{\mathrm{s}}\right)$ on the linear flake size $N$. Due to mirror symmetries of the unit cell, linear corrections do not appear for the shear parameter $\mu_{\mathrm{el}}$. Right: extrapolating the curvature into the bulk limit. curvature in this quantity, $\mu$, and how it evolves with the flake size. Since $\Delta E_{\mathrm{s}}$ is even in the shear strain, only positive values of $\varepsilon_{\mathrm{s}}$ are given. Also, for the same reason anharmonic terms exist only in the quartic order so that the displayed data traces have zero slope. Similar to the previous case of isotropic strain, we also witness here a very strong dependency of the elastic constant on the flake size. In fact, for shear strain it reaches almost $70 \%$ for the small system sizes that we are considering.

\section{Buckling-induced strain}

We present results from an additional DFT study, where we investigate the transverse stiffness of the graphene flake that gives rise to the elastic parameter $\kappa$. To this end we employ the following strategy. Each flake has a center pair or center ring of carbon atoms, see Figs. 1 and 8. To create a transverse probing field $h(\mathbf{r})$, we lift the center atoms by the distance $h_{0}$ over the reference plane. After this, the atomic
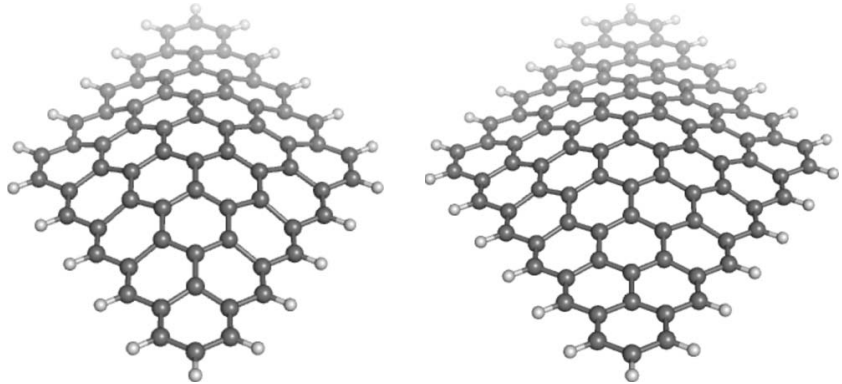

FIG. 8. Buckling flakes of $N=5$ and 6 with different central configurations of carbon atoms. The atomic configuration of $\mathrm{C}$ atoms is relaxed under the constraint that the center atoms remain at a given height $h_{0}$ above the ground plane while edge atoms ( $\mathrm{H}$ and C) remain sitting within this plane $(h=0)$. 


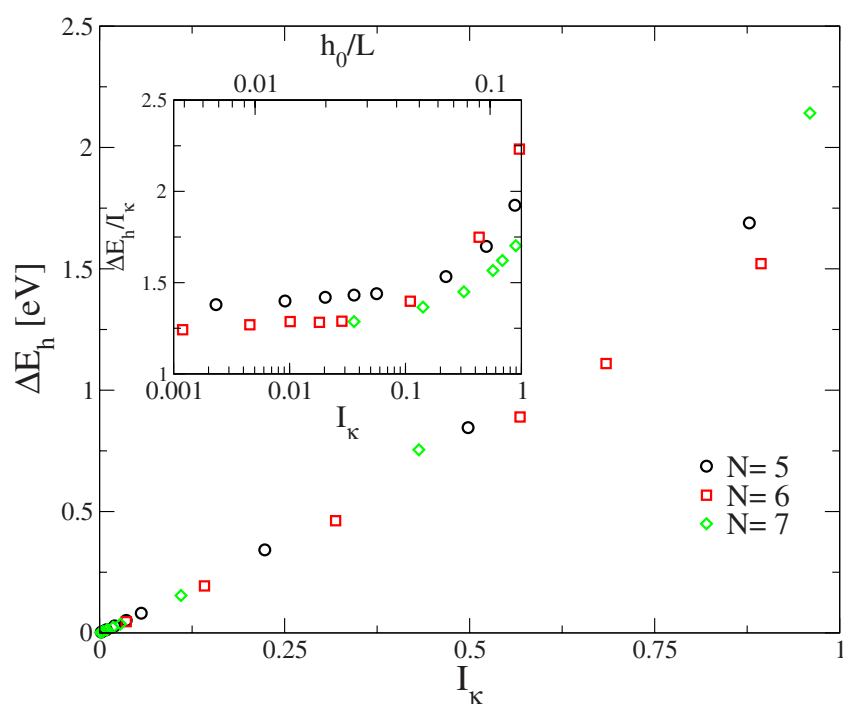

FIG. 9. (Color online) Estimating the Lamé parameter $\kappa$ determined from the excess energy $\Delta E$ of a bulging flake with maximum height at $h_{0}$ over the unperturbed (flat) plane (see Fig. 8). Main panel: change in energy $\Delta E$ with the integrated curvature $I_{\kappa}$ $=\int d^{2} \mathbf{r}[\Delta h(\mathbf{r})]^{2}$ for different flake sizes $N$. Inset: the ratio $\kappa$ $=\Delta E / I_{\kappa}$ depend on $N$ due to the effect of edge compression.

structure of the flake is relaxed under the constraint that the set of edge atoms ( $\mathrm{H}$ atoms and edge $\mathrm{C}$ atoms) can move only within the reference plane; edge atoms cannot shift in $h$ direction. ${ }^{77}$ In this way, a flake is equipped with a single ripple while at the same time the associated strain field $\mathbf{u}(\mathbf{x})$ remains negligibly small. In order to estimate the integrated curvature we numerically compute the bivariate function which interpolates the scattered data values $[h(\mathbf{r})$ field $]$ at any predefined smooth mesh. We then use this interpolated function to perform the second-order numerical derivative at any arbitrary precision.

Figure 9 displays how the excess energy $\Delta E_{h}$ associated with the ripple grows with the increased integrated curvature,

$$
I_{\kappa}=\frac{1}{2} \int d^{2} \mathbf{r}[\Delta h(\mathbf{r})]^{2} .
$$

The increase is linear, as expected from Eq. (2) with a slope that is only weakly dependent on the flake size, see inset Fig. 9. This implies that nonlinearities remain small as long as the ratio of the ripples amplitude and wavelength, $h_{0} / L$, does not exceed $\sim 5 \%$. The bending rigidity thus found is $\kappa_{\mathrm{el}}$ $=1.24 \mathrm{eV}$ which is well consistent with the value $1.1 \mathrm{eV}$ obtained by Fasolino et al. ${ }^{30}$

Notice that there is a significant scattering of almost $20 \%$ in earlier theoretical estimates for $\kappa$ and derived quantities, see Table 2 in Ref. 78. Discrepancies appear because different theoretical techniques are being employed, e.g., empirical potentials $^{42}$ and density-functional theory but also because of modeling artifacts. For example, extracting $\kappa$ from the elastic energy of carbon nanotubes (radius $R$ ) requires a very careful extrapolation in $1 / R$. If subleading terms are ignored, there is a pronounced tendency for overestimation, e.g., $\kappa$ $=1.46 \mathrm{eV}$ in Ref. 72. These authors used nanotubes with smaller tube radius, hence bigger curvature, where nonlinear effects become important. We can check the bending rigidity using the same curvature in Fig. 9 (inset) as reported in Ref. 72 and find a reasonable agreement with their value.

\section{ZERO-POINT MOTION}

In this chapter we extend our analysis of flake elastic properties and take also the zero-point motion of the atom cores into account, that constitute the hexagonal lattice. Now, the energy acquires a second term,

$$
F(N, d)=F_{\mathrm{el}}(N, d)+F_{\mathrm{vib}}(N, d)
$$

with

$$
F_{\mathrm{vib}}=\frac{1}{2} \sum_{p} \hbar \omega_{p}(N, d)
$$

where $p$ labels all the flake's vibrational modes. The vibrational excess energy associated with stretching the flake reads

$$
\Delta F_{\mathrm{vib}}=\frac{\hbar}{2} \sum_{p} \omega_{p}(N, d)-\omega_{p}[N, d(N)],
$$

where $\omega[N, d(N)]$ denotes the vibration energies in the absence of strain and $d(N)$ the equilibrium bond length, see inset Fig. 5. Also $\Delta F_{\text {vib }}$ can be expanded in terms of the slow elastic modes,

$$
\Delta F_{\mathrm{vib}}[h, \mathbf{u}]=\frac{1}{2} \int_{\mathcal{A}} d^{2} \mathbf{r} \gamma_{h}[\Delta h(\mathbf{r})]^{2}-\int_{\mathcal{A}} d^{2} \mathbf{r} \sum_{i j} \gamma_{u}^{i j} u_{i j}(\mathbf{r})
$$

with expansion parameters $\gamma_{h}, \gamma_{u}$ that represent averages of Grüneisen parameters over all vibrational modes. Specifically, we have in isotropic media

$$
\gamma_{h}=\hbar \sum_{p} \omega_{p} \Gamma_{p}^{h}, \quad \gamma_{u}^{i i}=\hbar \sum_{p} \omega_{p} \Gamma_{p}^{i i}
$$

where $\Gamma_{p}^{h}=\partial \ln \omega_{p} / \partial h^{i i}$ and $\Gamma_{p}^{i i}=\partial \ln \omega_{p} / \partial \varepsilon^{i i}$. In a twodimensional sample ${ }^{79}$ with a mirror symmetry one expects $\gamma_{u}^{x y}=\gamma_{u}^{y x}=0$; the change in phonon frequencies should be even in the shear strain, $\varepsilon_{\mathrm{s}}$. Combining Eq. (17) with an expansion of $\Delta F_{\text {el }}$ in full analogy with Eq. (2) and after completing the square, we find,

$$
\begin{aligned}
\Delta F_{\mathrm{elvib}}(N, d)= & \frac{\kappa_{\mathrm{el}}+\gamma_{h}}{2} \int_{A} d^{2} \mathbf{r}\left(\nabla^{2} h\right)^{2}+\frac{\mu_{\mathrm{el}}+\lambda_{\mathrm{el}}}{2} \int_{A} d^{2} \mathbf{r} \\
& \times\left(u_{i i}-\frac{\gamma_{u}^{i i}}{\mu_{\mathrm{el}}+\lambda_{\mathrm{el}}}\right)^{2}+\frac{\mu_{\mathrm{el}}}{2} \int_{A} d^{2} \mathbf{r}\left(u_{x x}-u_{y y}\right)^{2} \\
& +u_{x y}^{2} .
\end{aligned}
$$

For clarity, we have indicated in this expression the bare electronic coefficients (i.e., with frozen atomic cores) by $\kappa_{\mathrm{el}}, \mu_{\mathrm{el}}, \lambda_{\mathrm{el}}$. Likewise, the displacement field $\mathbf{u}(\mathbf{x})$ is defined with respect to the optimum flake geometry-ignoring vibrational terms. 

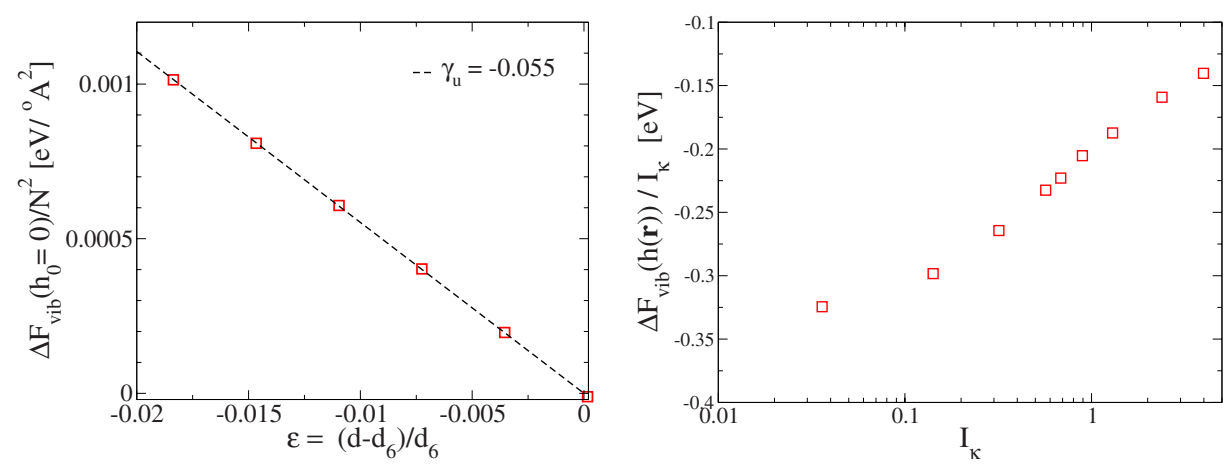

FIG. 10. (Color online) Left: dependency of $\Delta F_{v i b}$ on isotropic strain for system size $N=6$. The slope defines $\gamma_{u}$ as in Eq. (17). Right: dependency of $\Delta F_{v i b}$ on integrated curvature, $I_{K}=\int d^{2} \mathbf{r}$ $[\Delta h(\mathbf{r})]^{2}$, as in Fig. 9 .
In this way we can observe two facts. (i) Vibrations modify the bare transverse stiffness $\kappa_{\mathrm{el}}$ in Eq. (19), $\kappa=\kappa_{\mathrm{el}}$ $+\gamma_{h}$. (ii) Vibrations also affect interatomic distances. The effect can be understood as an effective strain, which stretches the bare C-C distances: $\gamma_{u}^{i i} /\left(\mu_{\mathrm{el}}+\lambda_{\mathrm{el}}\right)$. (iii) The change in the $\mathrm{C}-\mathrm{C}$ bond lengths eventually feeds back into all elastic coefficients. Therefore, in a more complete treatment of higher order terms also modifications in $\mu_{\mathrm{el}}, \lambda_{\mathrm{el}}$ would occur.

\section{A. DFT calculations of the Grüneisen parameters}

In order to estimate the Grüneisen parameters, $\gamma_{h}, \gamma_{u}$, we should calculate the vibrational spectrum of flakes with and without applied strain. To this end we adopt the following procedure. For every flake size, $N$, we find the atomic geometry with the optimal electronic energy, see, e.g., Fig. 1. This constitutes the set of freely relaxed "parent states." The relaxation ensures the Hessian, that characterizes interatomic forces, to become a positive definite matrix. ${ }^{80}$

In the present study we focus on the impact of phonons on the bulk elastic constants. There we may eliminate contributions of surface vibrations by assigning an infinite mass to the surface $\mathrm{H}$ and $\mathrm{C}$ atoms. Other than this, the calculation of vibrational modes and frequencies for the relaxed flake is a standard procedure. ${ }^{81,82}$

Thereafter, each parent state thus obtained is used in order to create two new families. The first family is constructed to obtain $\gamma_{u}$. It derives by changing the bond length of edge C-C-pairs by a factor of $1+\varepsilon$ keeping all atoms still inside the base plane $(h=0)$. For each value $\varepsilon$ the internal $\mathrm{C}$ atoms

TABLE VI. Survey over the fitted Grüneisen parameters extracted from the data in Figs. 10 and 11, respectively. For the definitions of $\gamma_{u, h}$ see Eq. (18).

\begin{tabular}{lccc}
\hline \hline & BP86, $N=6$ & Prev. calc. & Expt. \\
\hline$\gamma_{u}\left(\mathrm{eV} / \AA^{2}\right)$ & -0.055 & & \\
$\gamma_{h}(\mathrm{eV})$ & -0.32 & & \\
$\gamma_{u} /\left(\mu_{\mathrm{el}}+\lambda_{\mathrm{el}}\right)$ & -0.004 & & \\
$\gamma_{\mathrm{D}}$ & 2.6 & $2.7^{\mathrm{a}}$ & \\
$\gamma_{\mathrm{G}}$ & 2.2 & $2.0^{\mathrm{b}}$ & $1.99^{\mathrm{c}, \mathrm{d}}$ \\
\hline \hline
\end{tabular}

a Reference 70 .

${ }^{b}$ Reference 83.

'Reference 49.

${ }^{\mathrm{d}}$ Reference 53 . are relaxed and the vibrational spectrum together with the average strain field, $I_{u}(\varepsilon)=\int d \mathbf{r}\left(u_{x x}+u_{y y}\right)$, are recalculated. In this process it is important to have edge atoms at infinite mass. This ensures that the flake energy is in a (constrained) minimum so that all frequencies are real.

In order to determine $\gamma_{h}$ a second family has been constructed. It consists of the buckled flakes, Fig. 8, that we have studied in the previous section in order to extract $\kappa_{\mathrm{el}}$. Again, after assigning infinite mass to the edge atoms for each family member, the vibrational spectrum and the consecutive modification of the zero-point energy can be calculated.

\section{B. Results and discussion}

In Fig. 10 the change in the zero-point energy, $\Delta F_{\text {vib }}$, is plotted over the integrated strain fields. The Grüneisen parameters are given by the slope near zero strain; their numerical values are listed in Table VI. For a discussion of our results we first recall that the vibrational spectral density of states of the carbon sheet has a strong peak in the optical frequency regime, cf. Fig. 11, near $1600 \mathrm{~cm}^{-1}$. It is the "Gpeak," that corresponds to an in-plane mode, where neighboring atoms vibrate in opposite direction as depicted in Fig.
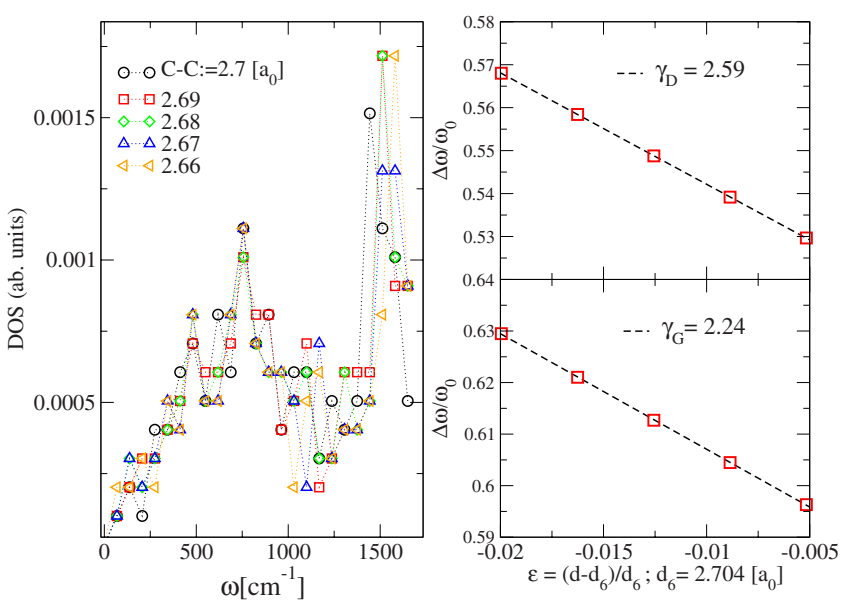

FIG. 11. (Color online) Left panel: phonon density of states for the flake of size $N=6$. Data sets are shown for different $\mathrm{C}-\mathrm{C}$ bond distances. Upper right panel shows the flow of D-peak with in-plane strain. Lower right panel shows the variation in Raman G-peak with strain, where $\omega_{0}$ is the Raman band frequency $\left(\approx 2680 \mathrm{~cm}^{-1}\right)$ (Ref. 52). The corresponding vibration modes are shown in Fig. 12. 

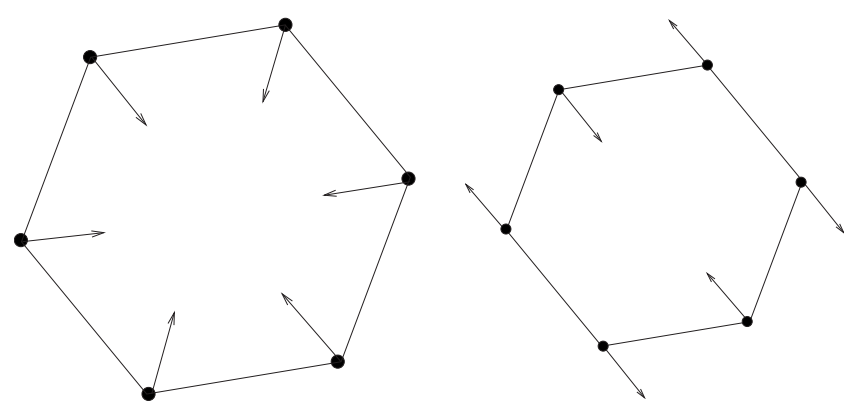

FIG. 12. Schematic of vibrating modes that give rise to D(left) and G(right) peaks in Raman spectra of graphene.

12 (right). This mode gives the dominating contribution to the total zero-point energy. Another significant contribution comes from the frequency range 500-1000 $\mathrm{cm}^{-1}$, where one observes the mixing of out-of-plane modes with in-plane modes. A third important mode is the "D-peak" near $1350 \mathrm{~cm}^{-1}$ that reflects the breathing mode as shown in Fig. 12 (left). This mode is particularly interesting when studying finite-size (edge) effects in graphene flakes. The reason is that in bulk graphene the coupling of the D-peak to the electromagnetic fields is suppressed, since the $D_{6 h}$ symmetry of the hexagonal unit cell remains intact and inhibits the formation of the dipole moment. By contrast, in flake with an overall symmetry that is lower than $D_{6 h}$, the D-peak is observable with a strength proportional to the inverse flake size.

Therefore, we can understand the sign of $\gamma_{h}$ as a consequence of a softening of these modes in the sample regions with nonzero curvature $[\Delta h(\mathbf{r})]^{2}$. Similarly, $\gamma_{u}$ is negative, indicating the increase in the atomic oscillator frequency that occurs when the interatomic distance is diminished.

While the sign of $\gamma_{h, u}$ was not unexpected, it is worth noting that the vibrational contributions to the phenomenological material parameters are actually not so small. The bare electronic bending rigidity, $\kappa_{\mathrm{el}}$ is reduced by as much as $26 \%$ down to $\kappa=\kappa_{\mathrm{el}}+\gamma_{h}=0.88 \mathrm{eV}$. Similarly, when expressing the effect of vibrations on the atomic lattice as an effective strain pushing the atoms to larger distances, then this strain reaches values up to $0.4 \%$.

Here, we also calculate the Grüneisen parameters associated with individual modes (see Fig. 12). The right panel in Fig. 11 shows the flow of the Raman frequencies (upper half $\mathrm{D}$, lower $\mathrm{G}$ ) with the applied strain. The frequency decreases linearly [as described in Eq. (17)] with decreasing compression due to anharmonicity of the interatomic potential. The slope essentially estimates the Grüneisen parameters, $\gamma_{\mathrm{G}}$ and $\gamma_{\mathrm{D}}$ for the vibrations shown in Fig. 12. Our results for $\gamma_{\mathrm{G}}$ and $\gamma_{\mathrm{D}}$ are consistent with the earlier experiments and first-principles calculations (see Table VI) available in literature. ${ }^{49,53,70,83}$

\section{CONCLUSION}

The elastic properties of edge-hydrogenated graphene flakes have been investigated employing the densityfunctional theory (DFT). Our study emphasizes the interplay between the edge and bulk properties which are mediated via long-range elastic forces.

Specifically, we are able to disentangle bulk, surface and corner contributions to the energy together with the leading higher order corrections. The binding energy per surface (edge) bond $(7.5 \mathrm{eV})$ is roughly $2 \mathrm{eV}$ higher than the one for interior (bulk) bonds $(5.2 \mathrm{eV})$; similarly, edge bonds have a tendency to be shorter than bulk ones. As a consequence, the flake's interior undergoes a surface-induced compression which is the more pronounced the smaller the flake is. This compression manifests itself in the way in which various observables depend on the flake size, $N$. For example, elastic constants (i.e., Lamé parameters) of small flakes can exceed their bulk limit $(\mu+\lambda \approx 70 \mathrm{eV}$ per ring, $\mu \approx 51 \mathrm{eV}$ per ring, $\nu=0.162)$ by $30 \%(\mu+\lambda)$ or even by $70 \%(\mu)$. In comparison, the sheet (out-of-plane, buckling) stiffness, $\kappa \approx 1.2 \mathrm{eV}$, is less sensitive to $N$. Nonlinearities remain weak (less than $10 \%$ increase) as long as the ratio of out-of-plane amplitude and in-plane wavelength of buckling is below 5\%. To highlight the importance of quantum effects on elasticity we have also calculated the vibrational spectrum of graphene flakes. Quantum corrections affect mostly the sheet stiffness, $\kappa$, lowering it significantly, about $26 \%$ within our DFT framework.

Finally, based on these results we predict a pronounced shift of the Raman G- and D-peaks with decreasing flake size to higher values. It is a natural consequence of the edgeinduced flake compression. The associated Grüneisen parameters are $\gamma_{\mathrm{D}} \approx 2.6$ and $\gamma_{\mathrm{G}} \approx 2.2$.

\section{ACKNOWLEDGMENTS}

We are grateful to A. Bagrets, M. van Setten, and V. Meded for helping us with the data visualization and also with the $a b$ initio calculation. We are thankful to $\mathrm{J}$. Weissmüller for numerous discussions during this work. Also, we acknowledge support from the Landesgraduiertenförderung Baden-Württemberg and Center of Functional Nanostructures of the Deutsche Forschungsgemeinschaft, project 4.11 .

\section{APPENDIX: COMPARISON}

In order to compare our results with previous authors here we perform a consistency check by evaluating the bond energies. We have a binding energy $\Psi\left(d_{0}\right)=-5.22 \mathrm{eV}$ for bulk carbon bonds. It translates into an energy per bulk $\mathrm{C}$ atom (cohesive energy) $3 \Psi\left(d_{0}\right) / 2 \approx-8.06 \mathrm{eV}$ which compares favorably well with an earlier estimate ${ }^{84}-7.9 \mathrm{eV}$.

For the edge energy we use the earlier definition, ${ }^{68,69}$

$$
E_{\text {edge }}=\frac{1}{L}\left(E_{\text {tot }}-N_{\mathrm{C}} E_{\mathrm{C}}^{\mathrm{b}}-\frac{N_{\mathrm{H}}}{2} E_{\mathrm{H}_{2}}\right),
$$

where $N_{C}$ is the total number of carbon atoms and $E_{C}^{\mathrm{b}}$ denotes the energy of a carbon atom in bulk graphene. $E_{\mathrm{H}_{2}}=$ $-31.496 \mathrm{eV}$ is the energy of a hydrogen molecule and $N_{\mathrm{H}}$ is the total number of hydrogen atoms that terminate the graphene edge of total length $L$. In order to estimate $E_{C}^{\mathrm{b}}$ we parameterize the total energy in full analogy with Eq. (1), 


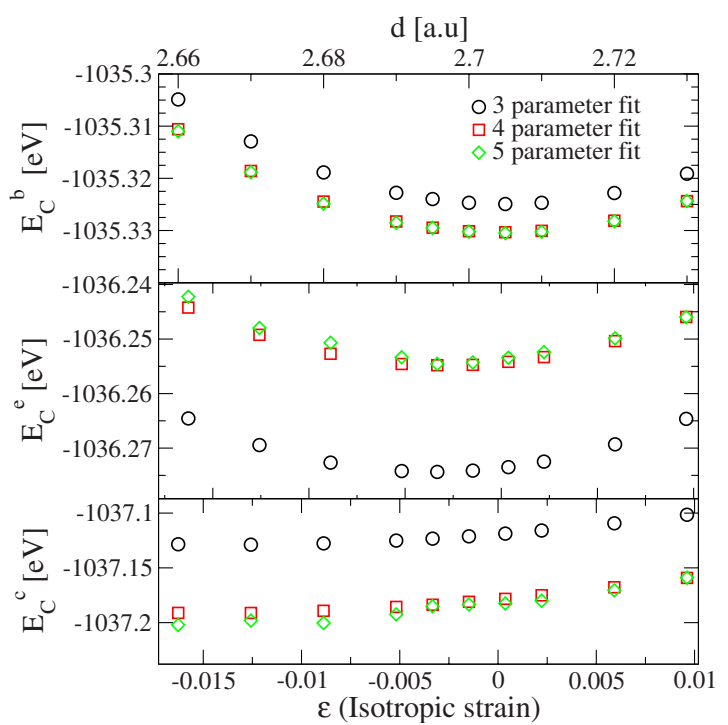

FIG. 13. (Color online) Bulk $\left(E_{\mathrm{C}}^{\mathrm{b}}\right)$, surface (edge, $\left.E_{\mathrm{C}}^{\mathrm{e}}\right)$, and corner $\left(E_{\mathrm{C}}^{\mathrm{c}}\right)$ energy as extracted from Eq. (A2) using density-functional theory (PBE functional). Databased on the evaluation of flake sizes ranging from $\{N\}=4, \ldots, 8$ per $d$ values $\left(a_{0}:=0.529 \AA\right.$ A.). (○) denotes data from a three-parameter fit to first three terms of Eq. (A2) ignoring the correction terms. $(\square)$ and $(\diamond)$ are the data with fourand five-parameter fits of Eq. (A2) including the correction terms. The scattering between the data sets that include the finite-size correction terms is relatively small, which shows the stability of the fitting procedure.

$$
E_{\mathrm{tot}}=N_{\mathrm{C}}^{\mathrm{b}} E_{\mathrm{C}}^{\mathrm{b}}+N_{\mathrm{C}}^{\mathrm{e}} E_{\mathrm{C}}^{\mathrm{e}}+N_{\mathrm{C}}^{\mathrm{c}} E_{\mathrm{C}}^{\mathrm{c}}+N_{\mathrm{H}} E_{\mathrm{H}}+\frac{\phi^{\mathrm{e}}}{N_{\mathrm{C}}^{\mathrm{e}}}+\frac{\phi^{\mathrm{b}}}{N_{\mathrm{C}}^{\mathrm{b}}}
$$

with

$$
N_{\mathrm{C}}=N_{\mathrm{C}}^{\mathrm{b}}+N_{\mathrm{C}}^{\mathrm{e}}+N_{\mathrm{C}}^{\mathrm{c}}
$$

where $N_{\mathrm{C}}^{\mathrm{b}}$ denotes the number of bulk carbon atoms (having only $\mathrm{C}$ atoms as nearest and next-nearest neighbors) with an associated energy $E_{\mathrm{C}}^{\mathrm{b}}, N_{\mathrm{c}}^{\mathrm{e}}$ denotes the number of edge carbon atoms with energy $E_{\mathrm{C}}^{\mathrm{e}}$, and $N_{\mathrm{C}}^{\mathrm{c}}$ is the number of corner carbon atoms with energy $E_{\mathrm{C}}^{\mathrm{c}}(\mathrm{CH}$ groups with at least one more $\mathrm{CH}$ group as nearest neighbor; $N_{\mathrm{C}}^{\mathrm{c}}=10$ in our case). $\phi^{\text {b,e }}$ are further expansion coefficients. $N_{\mathrm{H}}=\left(N_{\mathrm{C}}^{\mathrm{e}}-4\right) / 2+N_{\mathrm{C}}^{\mathrm{c}}$ is the total number of hydrogen atoms with energy per atom $E_{\mathrm{H}}=$ $-13.568 \mathrm{eV}$ in vacuum which is calculated separately. In order to estimate the bulk carbon energy we have performed a three-, four-, and five-parameter fit on the raw DFT data. The fit is stable against the number of fitting parameters with data set consisting of $N=4, \ldots, 8$, as shown in Fig. 13. Our estimate of the bulk energy $E_{C}^{\mathrm{b}}$, edge energy $E_{C}^{\mathrm{e}}$, and corner energy $E_{C}^{\mathrm{c}}$ per carbon atom are listed in Table VII.

Finally, using these estimates we calculate the edge energy per unit length and its variation with homogenous, isotropic strain, $\varepsilon$, (Fig. 14). The result at zero strain, $0.114 \pm 0.001 \mathrm{eV} / \AA$, compares well with earlier works $0.106 \mathrm{eV} / \AA .{ }^{68,69}$ Furthermore, we extract the edge stress, $\tau_{\mathrm{e}}$, following the conventional definition, ${ }^{68}$

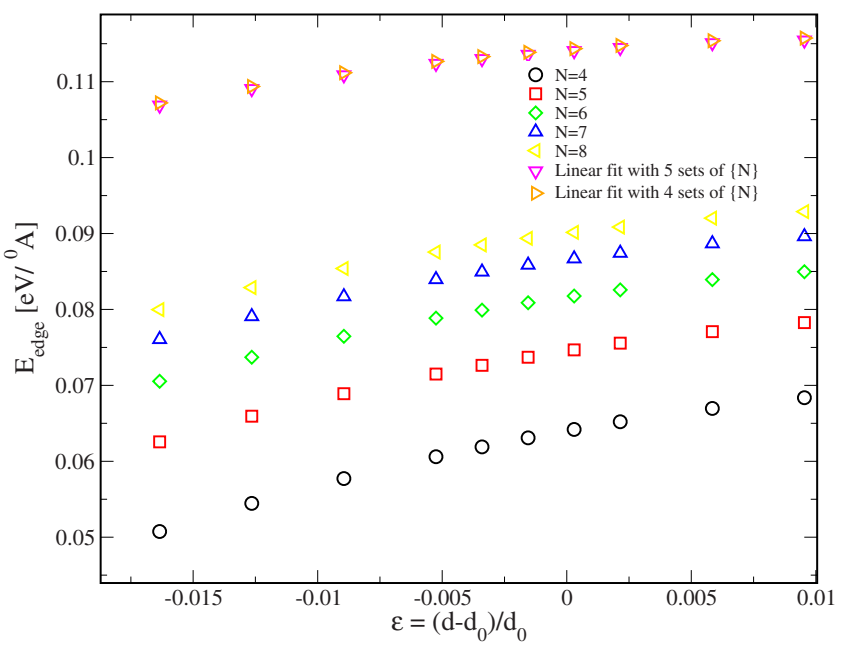

FIG. 14. (Color online) Change in the edge energy $\left(E_{\text {edge }}\right)$ as defined in Eq. (A1) when applying homogenous, isotropic strain $(\varepsilon)$ in graphene flakes). Data sets are calculated using PBE functional for system sizes $\{N\}=4, \ldots, 8 . d_{0}=1.431 \AA$, see, e.g., Table I. $(\nabla)$ and $(\triangleright)$ show the extrapolated value of $E_{\text {edge }}$ at $N_{\mathrm{C}}^{\mathrm{c}} / L \rightarrow 0$ limit per $d$ value using five $(\{N\}=4, \ldots, 8)$ and four system sizes $(\{N\}$ $=5, \ldots, 8)$, respectively. Almost no scattering between these two data sets illustrates the convergence of the fit.

$$
\tau_{\mathrm{e}}=\frac{d E_{\mathrm{edge}}}{d \varepsilon}
$$

Our value, $\tau_{\mathrm{e}}=0.26 \mathrm{eV} / \AA$, is significantly enhanced compared to the values reported for uniaxial strain, $\tau_{\mathrm{e}}^{\mathrm{ua}}=$ $-0.01 \mathrm{eV} / \AA,{ }^{68,69}$ that have been obtained in a ribbon geometry. The lower sensitivity on homogenous uniaxial strain is not a surprise, since in that case a considerable part of the elastic energy cost for longitudinal stretching can be released by the transverse contraction. With homogenous isotropic strain the surface unit cell experiences stretching in all directions and there is no partially compensating relaxation process.

In order to understand the relative smallness of the edge energy, $E_{\text {edge }}$, of the hydrogen-terminated graphene flakes we rewrite Eq. (A1) after substituting $E_{\text {tot }}$ from Eq. (A2),

TABLE VII. Survey over the fitted total-energy parameters and edge energy as defined in Eqs. (A1) and (A2). Data extracted at zero strain, where the bulk C-C bond distance is minimum, $d_{0}$ $=1.431 \AA$ (see Table I).

\begin{tabular}{lcc}
\hline \hline & PBE functional & Prev. calc. \\
\hline$E_{c}^{\mathrm{b}}(\mathrm{eV})$ & $-1035.33 \pm 0.01$ & \\
$E_{c}^{\mathrm{e}}(\mathrm{eV})$ & $-1036.25 \pm 0.02$ & \\
$E_{c}^{\mathrm{c}}(\mathrm{eV})$ & $-1037.18 \pm 0.06$ & \\
$E_{\text {edge }}(\mathrm{eV} / \AA)$ & $0.114 \pm 0.001$ & $0.106^{\mathrm{a}, \mathrm{b}}$ \\
\hline \hline
\end{tabular}

Reference 68 .

${ }^{\mathrm{b}}$ Reference 69 . 


$$
\begin{aligned}
E_{\text {edge }}= & \frac{N_{\mathrm{C}}^{\mathrm{e}}}{L}\left(E_{\mathrm{C}}^{\mathrm{e}}-E_{\mathrm{C}}^{\mathrm{b}}\right)+\frac{N_{\mathrm{C}}^{\mathrm{c}}}{L}\left(E_{\mathrm{C}}^{\mathrm{c}}-E_{\mathrm{C}}^{\mathrm{b}}\right)+\frac{1}{2} \frac{N_{\mathrm{H}}}{L} E_{\mathrm{H}_{2}}^{\mathrm{b}}+\frac{\phi^{\mathrm{e}}}{L N_{\mathrm{C}}^{\mathrm{e}}} \\
& +\frac{\phi^{\mathrm{b}}}{L N_{\mathrm{C}}^{\mathrm{b}}}
\end{aligned}
$$

with

$$
L=\sqrt{3} d \frac{N_{\mathrm{C}}^{\mathrm{e}}+4}{2}+\frac{8 d}{\sqrt{3}},
$$

where $N_{\mathrm{C}}^{\mathrm{e}}$ denotes the total number of edge $\mathrm{C}$ atoms of a graphene flake and $E_{\mathrm{H}_{2}}^{\mathrm{b}}=2 E_{\mathrm{H}}-E_{\mathrm{H}_{2}} \approx 4.36 \mathrm{eV}$ is the binding energy of a hydrogen atom. Now, considering the limit $L$ $\rightarrow \infty$, Eq. (A5) reduces to

$$
\lim _{L \rightarrow \infty} E_{\text {edge }}=\frac{E_{\mathrm{C}}^{\mathrm{e}}-E_{\mathrm{C}}^{\mathrm{b}}+E_{\mathrm{H}_{2}}^{\mathrm{b}} / 4}{\sqrt{3} d / 2} .
$$

Using the above-mentioned estimate, $E_{\mathrm{C}}^{\mathrm{e}}-E_{\mathrm{C}}^{\mathrm{b}} \approx \approx-0.92$, we see that difference in bulk and edge binding energy is largely compensated by the additional binding energy averaged over two edge $\mathrm{C}$ atoms contributed by the $\mathrm{H}_{2}$ molecule, $E_{\mathrm{H}_{2}}^{\mathrm{b}} / 4$ $\approx 1.09$.
${ }^{1}$ K. S. Novoselov, A. K. Geim, S. V. Morozov, D. Jiang, Y. Zhang, S. V. Dubonos, I. V. Grigorieva, and A. A. Firsov, Science 306, 666 (2004).

${ }^{2}$ Y. Zhang, Y.-W. Tan, H. L. Stormer, and P. Kim, Nature (London) 438, 201 (2005).

${ }^{3}$ K. S. Novoselov, E. McCann, S. V. Morozov, V. I. Fal'ko, M. I. Katsnelson, U. Zeitler, D. Jiang, F. Schedin, and A. K. Geim, Nat. Phys. 2, 177 (2006).

${ }^{4}$ A. K. Geim and K. S. Novoselov, Nature Mater. 6, 183 (2007).

${ }^{5}$ K. S. Novoselov, D. Jiang, F. Schedin, T. J. Booth, V. V. Khotkevich, S. V. Morozov, and A. K. Geim, Proc. Natl. Acad. Sci. U.S.A. 102, 10451 (2005).

${ }^{6}$ A. H. Castro Neto, F. Guinea, N. M. R. Peres, K. S. Novoselov, and A. K. Geim, Rev. Mod. Phys. 81, 109 (2009).

${ }^{7}$ K. Nakada, M. Fujita, G. Dresselhaus, and M. S. Dresselhaus, Phys. Rev. B 54, 17954 (1996).

${ }^{8}$ C. Stampfer, J. Güttinger, S. Hellmüller, F. Molitor, K. Ensslin, and T. Ihn, Phys. Rev. Lett. 102, 056403 (2009).

${ }^{9}$ M. Fujita, K. Wakabayashi, K. Nakada, and K. Kusakabe, J. Phys. Soc. Jpn. 65, 1920 (1996).

${ }^{10}$ L. Brey and H. A. Fertig, Phys. Rev. B 73, 235411 (2006).

${ }^{11}$ K. Wakabayashi, M. Sigrist, and M. Fujita, J. Phys. Soc. Jpn. 67, 2089 (1998).

${ }^{12}$ Y.-W. Son, M. L. Cohen, and S. G. Louie, Nature (London) 444, 347 (2006)

${ }^{13}$ Y.-W. Son, M. L. Cohen, and S. G. Louie, Phys. Rev. Lett. 97, 216803 (2006).

${ }^{14}$ O. Hod, V. Barone, J. E. Peralta, and G. E. Scuseria, Nano Lett. 7, 2295 (2007).

${ }^{15}$ E.-j. Kan, Z. Li, J. Yang, and J. G. Hou, J. Am. Chem. Soc. 130, 4224 (2008).

${ }^{16}$ N. M. R. Peres, A. H. Castro Neto, and F. Guinea, Phys. Rev. B 73, 195411 (2006)

${ }^{17}$ L. Pisani, J. A. Chan, B. Montanari, and N. M. Harrison, Phys. Rev. B 75, 064418 (2007).

${ }^{18}$ D.-e. Jiang, B. G. Sumpter, and S. Dai, J. Chem. Phys. 127, 124703 (2007).

${ }^{19}$ J. Fernández-Rossier and J. J. Palacios, Phys. Rev. Lett. 99, 177204 (2007).

${ }^{20}$ W. L. Wang, O. V. Yazyev, S. Meng, and E. Kaxiras, Phys. Rev. Lett. 102, 157201 (2009).

${ }^{21}$ C. Gómez-Navarro, M. Burghard, and K. Kern, Nano Lett. 8, 2045 (2008).
${ }^{22}$ R. Danneau, F. Wu, M. F. Craciun, S. Russo, M. Y. Tomi, J. Salmilehto, A. F. Morpurgo, and P. J. Hakonen, Phys. Rev. Lett. 100, 196802 (2008).

${ }^{23}$ P. Lauffer, K. V. Emtsev, R. Graupner, T. Seyller, L. Ley, S. A. Reshanov, and H. B. Weber, Phys. Rev. B 77, 155426 (2008).

${ }^{24}$ X. Li, X. Wang, L. Zhang, S. Lee, and H. Dai, Science 319, 1229 (2008).

${ }^{25}$ M. Haluška et al., Phys. Status Solidi B 244, 4143 (2007).

${ }^{26}$ F. Miao, S. Wijeratne, Y. Zhang, U. C. Coskun, W. Bao, and C. N. Lau, Science 317, 1530 (2007).

${ }^{27}$ C. Casiraghi, A. Hartschuh, H. Qian, S. Piscanec, C. Georgi, A. Fasoli, K. S. Novoselov, D. M. Basko, and A. C. Ferrari, Nano Lett. 9, 1433 (2009).

${ }^{28}$ A. L. Vázquez de Parga, F. Calleja, B. Borca, M. C. G. Passeggi, Jr., J. J. Hinarejos, F. Guinea, and R. Miranda, Phys. Rev. Lett. 100, 056807 (2008)

${ }^{29}$ C. Tan, Z. Tan, K. Wang, L. Ma, F. Yang, F. Qu, J. Chen, C. Yang, and L. Lu, arXiv:0910.5777 (unpublished).

${ }^{30}$ A. Fasolino, J. H. Los, and M. I. Katsnelson, Nature Mater. 6, 858 (2007)

${ }^{31}$ G. Gui, J. Li, and J. Zhong, Phys. Rev. B 78, 075435 (2008).

${ }^{32}$ J. Atalaya, A. Isacsson, and J. M. Kinaret, Nano Lett. 8, 4196 (2008).

${ }^{33}$ D. Garcia-Sanchez, A. M. van der Zande, A. S. Paulo, B. Lassagne, P. L. McEuen, and A. Bachtold, Nano Lett. 8, 1399 (2008).

${ }^{34}$ C. Lee, X. Wei, J. W. Kysar, and J. Hone, Science 321, 385 (2008).

${ }^{35}$ V. M. Pereira, A. H. Castro Neto, and N. M. R. Peres, Phys. Rev. B 80, 045401 (2009).

${ }^{36}$ S.-M. Choi, S.-H. Jhi, and Y.-W. Son, Phys. Rev. B 81, 081407 (2010).

${ }^{37}$ K. V. Zakharchenko, M. I. Katsnelson, and A. Fasolino, Phys. Rev. Lett. 102, 046808 (2009).

${ }^{38}$ O. Hod and G. E. Scuseria, Nano Lett. 9, 2619 (2009).

${ }^{39}$ E. Cadelano, P. L. Palla, S. Giordano, and L. Colombo, Phys. Rev. Lett. 102, 235502 (2009).

${ }^{40}$ F. Guinea, B. Horovitz, and P. Le Doussal, Solid State Commun. 149, 1140 (2009).

${ }^{41}$ C. D. Reddy, A. Ramasubramaniam, V. B. Shenoy, and Y.-W. Zhang, Appl. Phys. Lett. 94, 101904 (2009).

${ }^{42}$ Q. Lu, M. Arroyo, and R. Huang, J. Phys. D: Appl. Phys. 42, 102002 (2009). 
${ }^{43}$ R. M. Ribeiro, V. M. Pereira, N. M. R. Peres, P. R. Briddon, and A. H. Castro Neto, New J. Phys. 11, 115002 (2009).

${ }^{44}$ J. C. Meyer, A. K. Geim, M. I. Katsnelson, K. S. Novoselov, T. J. Booth, and S. Roth, Nature (London) 446, 60 (2007).

${ }^{45}$ E. Stolyarova, K. Rim, S. Ryu, J. Maultzsch, P. Kim, L. Brus, T. Heinz, M. Hybertsen, and G. Flynn, Proc. Natl. Acad. Sci. U.S.A. 104, 9209 (2007).

${ }^{46}$ M. Ishigami, J. H. Chen, W. G. Cullen, M. S. Fuhrer, and E. D. Williams, Nano Lett. 7, 1643 (2007).

${ }^{47}$ W. Bao, F. Miao, Z. Chen, H. Zhang, W. Jang, C. Dames, and C. N. Lau, Nat. Nanotechnol. 4, 562 (2009).

${ }^{48}$ T. Low and F. Guinea, Nano Lett. 10, 3551 (2010).

${ }^{49}$ T. M. G. Mohiuddin et al., Phys. Rev. B 79, 205433 (2009).

${ }^{50}$ C. C. Chen, W. Bao, J. Theiss, C. Dames, C. N. Lau, and S. B. Cronin, Nano Lett. 9, 4172 (2009).

${ }^{51}$ A. C. Ferrari et al., Phys. Rev. Lett. 97, 187401 (2006).

${ }^{52}$ Z. H. Ni, T. Yu, Y. H. Lu, Y. Y. Wang, Y. P. Feng, and Z. X. Shen, ACS Nano 2, 2301 (2008).

${ }^{53}$ J. E. Proctor, E. Gregoryanz, K. S. Novoselov, M. Lotya, J. N. Coleman, and M. P. Halsall, Phys. Rev. B 80, 073408 (2009).

${ }^{54}$ G. Tsoukleri, J. Parthenios, K. Papagelis, R. Jalil, A. C. Ferrari, A. K. Geim, K. S. Novoselov, and C. Galiotis, Small 5, 2397 (2009).

${ }^{55}$ S. Reich, H. Jantoljak, and C. Thomsen, Phys. Rev. B 61, R13389 (2000).

${ }^{56}$ D. Sánchez-Portal, E. Artacho, J. M. Soler, A. Rubio, and P. Ordejón, Phys. Rev. B 59, 12678 (1999).

${ }^{57}$ S. Reich, C. Thomsen, and P. Ordejón, Phys. Rev. B 65, 153407 (2002).

${ }^{58}$ O. Hod, J. E. Peralta, and G. E. Scuseria, Phys. Rev. B 76, 233401 (2007).

${ }^{59}$ L. Landau and E. Lifshitz, Theory of Elasticity (Pergamon Press, New York, 1959), Vol. 7

${ }^{60}$ Note that certain effects related to the thickness of the graphene sheet are left out in Eq. (2). For example, a linear displacement field $[h(\mathbf{r})=x, \mathbf{u}=0]$ has no energy cost because the description assumes that such a conformation is equivalent to a rotation. However, this ignores that a rotation gives the $\pi_{z}$ orbitals a new direction in space while the lifting up of atoms mediated by the linear displacement field does not. In the latter case, there is an additional energy cost $\propto|\nabla h|^{2}$ related to the fact that the overlap of $\pi_{z}$ orbitals changes, which is not included in Eq. (2). Similarly, global rotational invariance is given only for free flakes. It can be broken due to experimental boundary conditions, e.g., the attachment of contacts. Also this may produce gradient terms $(\nabla h)^{2}$ in the functional Eq. (2).
${ }^{61}$ R. Ahlrichs, M. Bär, M. Häser, H. Horn, and C. Kölmel, Chem. Phys. Lett. 162, 165 (1989).

${ }^{62}$ A. D. Becke, Phys. Rev. A 38, 3098 (1988).

${ }^{63}$ J. P. Perdew, Phys. Rev. B 33, 8822 (1986).

${ }^{64}$ J. P. Perdew and Y. Wang, Phys. Rev. B 45, 13244 (1992).

${ }^{65}$ J. P. Perdew, K. Burke, and M. Ernzerhof, Phys. Rev. Lett. 77, 3865 (1996).

${ }^{66}$ A. D. Becke, J. Chem. Phys. 98, 5648 (1993).

${ }^{67}$ A. Schäfer, H. Horn, and R. Ahlrichs, J. Chem. Phys. 97, 2571 (1992).

${ }^{68}$ C. K. Gan and D. J. Srolovitz, Phys. Rev. B 81, 125445 (2010).

${ }^{69}$ S. Jun, Phys. Rev. B 78, 073405 (2008).

${ }^{70}$ N. Mounet and N. Marzari, Phys. Rev. B 71, 205214 (2005).

${ }^{71}$ M. Arroyo and T. Belytschko, Phys. Rev. B 69, 115415 (2004).

${ }^{72}$ K. N. Kudin, G. E. Scuseria, and B. I. Yakobson, Phys. Rev. B 64, 235406 (2001).

${ }^{73}$ R. Faccio, P. A. Denis, H. Pardo, C. Goyenola, and A. W. Mombru, J. Phys.: Condens. Matter 21, 285304 (2009).

${ }^{74}$ R. C. Weast and M. J. Astle, CRC Handbook of Chemistry and Physics, 89th ed. (CRC Press, New York, 2009).

${ }^{75}$ O. L. Blakslee, D. G. Proctor, E. J. Seldin, G. B. Spence, and T. Weng, J. Appl. Phys. 41, 3373 (1970).

${ }^{76}$ A. Bosak, M. Krisch, M. Mohr, J. Maultzsch, and C. Thomsen, Phys. Rev. B 75, 153408 (2007).

${ }^{77}$ The motion of edge atoms in the plane is further restricted in the sense that only conformations have been considered, which can be obtained by a homogenous rescaling of all $\mathrm{C}-\mathrm{C}$ edge bond lengths.

${ }^{78}$ G. M. Odegard, T. S. Gates, L. M. Nicholson, and K. E. Wise, Compos. Sci. Technol. 62, 1869 (2002).

${ }^{79}$ Recall that due to the presence of edge the symmetry group of our sample is lower $\left(D_{2 h}\right)$ than the one of the unperturbed hexagonal unit cell $\left(D_{6 h}\right)$.

${ }^{80}$ This relaxation process is the limiting computational step. For a flake with $N=6$ it took several days on a single Opteron processor. For $N=6$ a single iteration takes about 1 day and for full convergence one needs typically 50 days.

${ }^{81}$ P. Deglmann, F. Furche, and R. Ahlrichs, Chem. Phys. Lett. 362, 511 (2002).

${ }^{82}$ P. Deglmann and F. Furche, J. Chem. Phys. 117, 9535 (2002).

${ }^{83}$ C. Thomsen, S. Reich, and P. Ordejón, Phys. Rev. B 65, 073403 (2002).

${ }^{84}$ P. Koskinen, S. Malola, and H. Häkkinen, Phys. Rev. Lett. 101, 115502 (2008) 